\title{
Automation of optimization of discrete technological processes
}

\author{
Evgeniy Kudryavtsev ${ }^{1, *}$ \\ ${ }^{1}$ Moscow State University of Civil Engineering, Yaroslavskoe shosse, 26, Moscow, 129337, Russia
}

\begin{abstract}
The article describes the procedure automation of optimization discrete technological processes with using of Bellman's functional (recurrent) equation and system Mathcad. As rule the technological processes includes $n$ of operations and each operation can be executed by various types of equipment. Expenses (cost, time, ...) on execution of $i$ operation by $k$ equipment after execution by $j$ equipment $(i-l)$ operation are known $-c(i, j, k)$. Expenses for execution by $k$ equipment $i$ operation can depend on the equipment $-j$, which executed previous $(i-1)$ operation. It is necessary to execute automation of optimization technological process with the minimum expenses. The algorithm of the decision of a problem by Bellman's method includes two phases. The first phase is calculations of the minimum expenses for execution of all partial technological processes, from last operation of process to the first. The second phase is definition of the required optimum set of equipment which is carrying out all technological process with the minimum expenses. The proposed procedure of automation of optimization technological process using Bellman's method and system Mathcad significantly decreases time and labour costs on execution of such calculations and efficiently to execute investigations related with change of equipment parameters.
\end{abstract}

\section{Introduction}

For execution automation of optimization of technological processes it's possible to use various decision methods and tools [1-20].

The technological process includes $n$ of operations. Each operation can be executed various types of equipment. Expenses (cost, time, ...) on execution of $i$ operation by $k$ equipment after execution by $j$ equipment $(i-1)$ operation are known $-c(i, j, k)$. Thus, the size of expenses for execution by $k$ equipment of $i$ operation can depend on the equipment $j$, execution previous $(i-1)$ operation. It is necessary to execute structurally-parametrical optimization of technological process with the minimum expenses.

The initial information is presented in the Table 1.

* Corresponding author: SDM@MGSU.RU 
Table 1. Initial information.

\begin{tabular}{|c|l|l|l|r|r|r|r|r|r|r|}
\hline \multirow{3}{*}{ Operation } & \multicolumn{8}{|c|}{ The equipment $\boldsymbol{j}$ executes previous (i-1) operation } \\
\cline { 2 - 11 } & \multicolumn{6}{|c|}{$j=1$} & \multicolumn{5}{|c|}{$j=2$} & \multicolumn{3}{|c|}{$j=3$} \\
\cline { 2 - 10 } & \multicolumn{7}{|c|}{ The equipment $\boldsymbol{k}$ executes flowing $\boldsymbol{i}$ operation } \\
\cline { 2 - 10 } & $k=1$ & $k=2$ & $k=3$ & $k=1$ & $k=2$ & $k=3$ & $k=1$ & $k=2$ & $k=3$ \\
\cline { 2 - 10 } & \multicolumn{7}{|c|}{ Expenses (cost, time, ..) $\boldsymbol{c}(\boldsymbol{i}, \boldsymbol{j}, \boldsymbol{k})$} \\
\hline 1 & 46 & 48 & 35 & - & - & - & - & - & - \\
\hline 2 & 157 & 150 & 145 & 155 & 150 & 146 & 165 & 162 & 160 \\
\hline 3 & 54 & 58 & - & 56 & 60 & - & 58 & 62 & \\
\hline 4 & 21 & 24 & - & 20 & 18 & - & - & - & - \\
\hline 5 & 0 & - & - & 0 & - & - & - & - & - \\
\hline
\end{tabular}

\section{Description of technological process}

Let's present all possible sets of equipment of the technological process in the form of the graph of the technological process figure 1. Each equipment is represented by a circle (Figure 1). The operation is represented in the form of an arrow with expenses $-c(i, j, k)$ on execution of $i$ operation by $k$ equipment after of $j$ equipment.

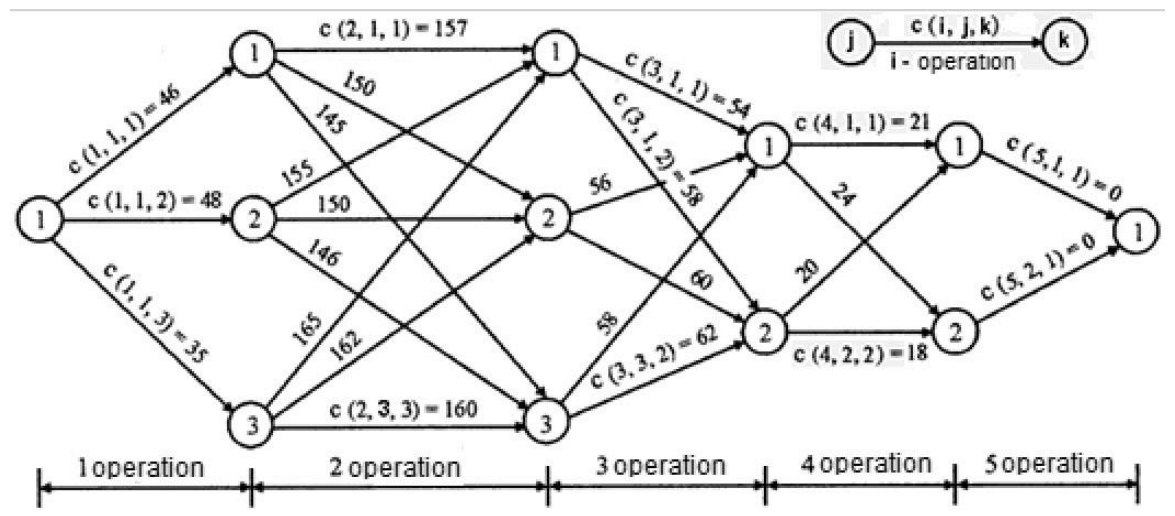

Fig. 1. Graph of discrete technological process

The circle (equipment) $j$ executes $(i-1)$ previous operation. The circle (equipment) $k$ executes (i) current operation.

Arrow is possible tie of one type of the equipment with other. The circle (equipment) also means execution of operation by one type of the equipment and the beginning of execution of other operation by other type of the equipment.

The number $j$ standing in the circle is type of the equipment with its parameters used on previous operation. The number $k$ standing in the circle is type of the equipment with its parameters used on current operation. First and last circles of the graph - fictitious equipment.

If at execution of any operation the subsequent equipment parameters mismatch previous equipment parameters then the given equipment cannot be used. In this case don't use an arrow between these equipment or use arrow with very big expenses so that this equipment could not be at the optimum set of the equipment. 
Representation of all possible sets of equipment in the form of the graph provides pictorial view and simplicity of formation of admissible set of structures of technological process.

\section{Mathematical model}

Original mathematical model is Bellman's recurrence equation for the problem decision:

$$
y(i, j)=\min _{k=1,2, \ldots, m_{i}}[c(i, j, k)+y(i+1, \mathrm{k})], \quad \mathrm{y}(6,1)=0,
$$

where: $y(i+1, k)$ - the minimum expenses for the partial sets equipment, carrying out partial technological process, from $i=(\mathrm{i}+1)$ till 1 operation and $k$ the equipment;

$y(i, j)$ - the same, from $i$ till $j=1$ operation and $j$-th equipment.

\section{Algorithm of the decision}

The algorithm of the decision of a problem by means of Bellman's equation includes two stages.

The first stage includes calculations of the minimum expenses for execution of all partial technological processes, since the last operation. Results of calculation are putting above or under a circle fig. 2. The arrow is directed from the equipment which has ensured the minimum expenses for considered partial technological process is marking by a stroke.

The second stage includes definition of the required optimum set of equipment which is carrying out all technological process with the minimum expenses.

The first stage includes several steps.

1. Calculation of the minimum expenses for execution of technological process including $i=5$ operation.

$$
y(5,1)=\min _{k=1}\left[\begin{array}{l}
c(5,1,1)+y(6,1) \\
c(5,2,1)+y(6,1)
\end{array}\right]=\min _{k=1}\left[\begin{array}{l}
0+0 \\
0+0
\end{array}\right]=0,
$$

Install by strokes on an arrow $(1,1)$ and an arrow $(2,1)$ of the 5 fictitious operation (see fig. 2) which has specified the equipment $j=1$ and $j=2$ which has ensured the minimum expenses.

2. Calculation of the minimum expenses for execution of 5-th and 4-th operations:

- the minimum expenses for partial technological process $y(4,1) /$

$$
y(4,1)=\min _{k=1,2}\left[\begin{array}{l}
c(4,1,1)+y(5,1) \\
c(4,1,2)+y(5,2)
\end{array}\right]=\min _{k=1,2}\left[\begin{array}{l}
21+0 \\
24+0
\end{array}\right]=21,
$$

Install stroke on an arrow (1-1) of the 4-th operation (see Figure 2) which have specified the equipment: $k=1$ ensured the minimum expenses for partial set of equipment.

- the minimum expenses for partial technological process $y(4,2)$

$$
y(4,2)=\min _{k=1,2}\left[\begin{array}{l}
c(4,2,1)+y(5,1) \\
c(4,2,2)+y(5,2)
\end{array}\right]=\min _{k=1,2}\left[\begin{array}{c}
20+0 \\
18+0
\end{array}\right]=18,
$$

Install stroke on an arrow (2-2) of the 4-th operation (see Figure 2) which have specified the equipment: $k=2$ ensured the minimum expenses for partial set of equipment. 
3. Calculation of the minimum expenses for execution of 5-th, 4-th and 3-th operations:

- the minimum expenses for partial technological process $y(3,1)$

$$
y(3,1)=\min _{k=1,2}\left\{\begin{array}{l}
c(3,1,1)+y(4,1) \\
c(3,1,2)+y(4,2)
\end{array}\right\}=\min _{k=1,2}\left\{\begin{array}{c}
54+21 \\
58+18
\end{array}\right\}=\min _{k=1,2}\left\{\begin{array}{l}
75 \\
76
\end{array}\right\}=75 .
$$

Install stroke on an arrow $(1,1)$ of the 3 -th operation (see Figure 2) which has specified the equipment $k=1$, ensured the minimum expenses.

- the minimum expenses for partial technological process $y(3,2)$

$$
y(3,2)=\min _{k=1,2}\left\{\begin{array}{c}
c(3,2,1)+y(4,1) \\
c(3,2,2)+y(4,2)
\end{array}\right\}=\min _{k=1,2}\left\{\begin{array}{l}
56+21 \\
60+18
\end{array}\right\}=\min _{k=1,2}\left\{\begin{array}{l}
77 \\
78
\end{array}\right\}=77
$$

Install stroke on an arrow (2,1) of the 3-th operation (see Figure 2) which has specified the equipment $k=1$, ensured the minimum expenses.

- the minimum expenses for partial technological process $y(3,3)$

$$
y(3,3)=\min _{k=1,2}\left\{\begin{array}{l}
c(3,3,1)+y(4,1) \\
c(3,3,2)+y(4,2)
\end{array}\right\}=\min _{k=1,2}\left\{\begin{array}{l}
58+21 \\
62+18
\end{array}\right\}=\min _{k=1,2}\left\{\begin{array}{l}
79 \\
80
\end{array}\right\}=79 .
$$

Install stroke on an arrow $(3,1)$ of the 3-th operation (see Figure 2) which has specified the equipment $k=1$, ensured the minimum expenses.

4. Calculation of the minimum expenses for execution of 5-th, 4-th, 3-th and 2-nd operations:

- the minimum expenses for partial technological process $y(2,1)$

$$
y(2,1)=\min _{k=1,2,3}\left\{\begin{array}{l}
c(2,1,1)+y(3,1) \\
c(2,1,2)+y(3,2) \\
c(2,1,3)+y(3,3)
\end{array}\right\}=\min _{k=1,2,3}\left\{\begin{array}{l}
157+75 \\
150+77 \\
145+79
\end{array}\right\}=\min _{k=1,2,3}\left\{\begin{array}{l}
232 \\
227 \\
224
\end{array}\right\}=224 .
$$

Install stroke on an arrow $(1,3)$ of the 2-nd operation (see Figure 2) which has specified the equipment $k=3$, ensured the minimum expenses.

- the minimum expenses for partial technological process $y(2,2)$

$$
y(2,2)=\min _{k=1,2,3}\left\{\begin{array}{l}
c(2,2,1)+y(3,1) \\
c(2,2,2)+y(3,2) \\
c(2,2,3)+y(3,3)
\end{array}\right\}=\min _{k=1,2,3}\left\{\begin{array}{l}
155+75 \\
150+77 \\
146+79
\end{array}\right\}=\min _{k=1,2,3}\left\{\begin{array}{l}
230 \\
227 \\
225
\end{array}\right\}=225 .
$$

Install stroke on an arrow $(2,3)$ of the 2-nd operation (see Figure 2) which has specified the equipment $k=3$, ensured the minimum expenses.

- the minimum expenses for partial technological process $y(2,3)$

$$
y(2,3)=\min _{k=1,2,3}\left\{\begin{array}{l}
c(2,3,1)+y(3,1) \\
c(2,3,2)+y(3,2) \\
c(2,3,3)+y(3,3)
\end{array}\right\}=\min _{k=1,2,3}\left\{\begin{array}{l}
165+75 \\
162+77 \\
160+79
\end{array}\right\}=\min _{k=1,2,3}\left\{\begin{array}{l}
240 \\
239 \\
239
\end{array}\right\}=239 .
$$


Install strokes on arrows $(3,2)$ and $(3,3)$ of the 2-nd operation (see Figure 2) which has specified the equipment $k=2$ and $k=3$, ensured the minimum expenses.

5. Calculation of the minimum expenses for execution of all operations:

- the minimum expenses for all technological process $y(1,1)$, which includes $1,2,3$ and 4 operations

$$
y(1,1)=\min _{k=1,2,3}\left\{\begin{array}{l}
c(1,1,1)+y(2,1) \\
c(1,1,1)+y(2,2) \\
c(1,1,3)+y(2,3)
\end{array}\right\}=\min _{k=1,2,3}\left\{\begin{array}{l}
46+224 \\
48+225 \\
35+239
\end{array}\right\}=\min _{k=1,2,3}\left\{\begin{array}{l}
270 \\
273 \\
274
\end{array}\right\}=270 .
$$

Install stroke on an arrow $(1,1)$ of the first operation (see Figure 2) which has specified the equipment $k=1$, ensured the minimum expenses.

Results of calculation are presented on Figure 2.

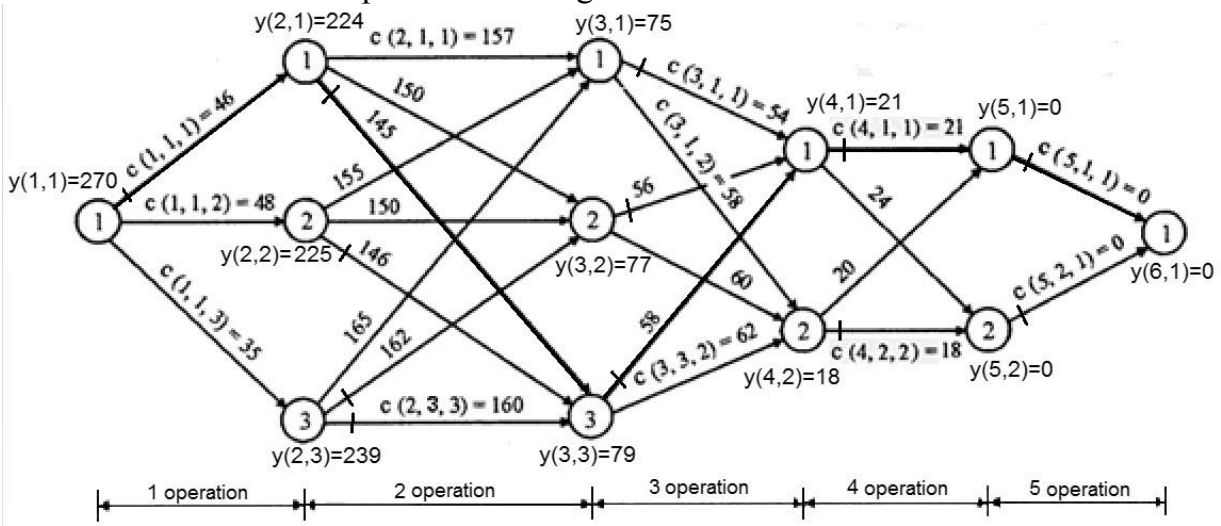

Fig. 2. Graph of the technological process with results of calculation

The second stage is carried out in reverse order, from search of the optimum equipment, carrying out last operation.

Thus, in our example in the optimum set of equipment (see thick lines) enter on: - 5-th (fictitious) operation the equipment under number $k=1$; 4-th operation the equipment under number $k=1$; 3 -rd operation the equipment under number $k=3$; 2-nd operations the equipment under number $k=1 ; 1$-st operation the equipment under number $k=1$.

\section{Automation of optimization in system Mathcad}

The algorithm and programs automation of optimization of technological process by Bellman's method conducted in system Mathcad [2-5] is given below Figure 3. 


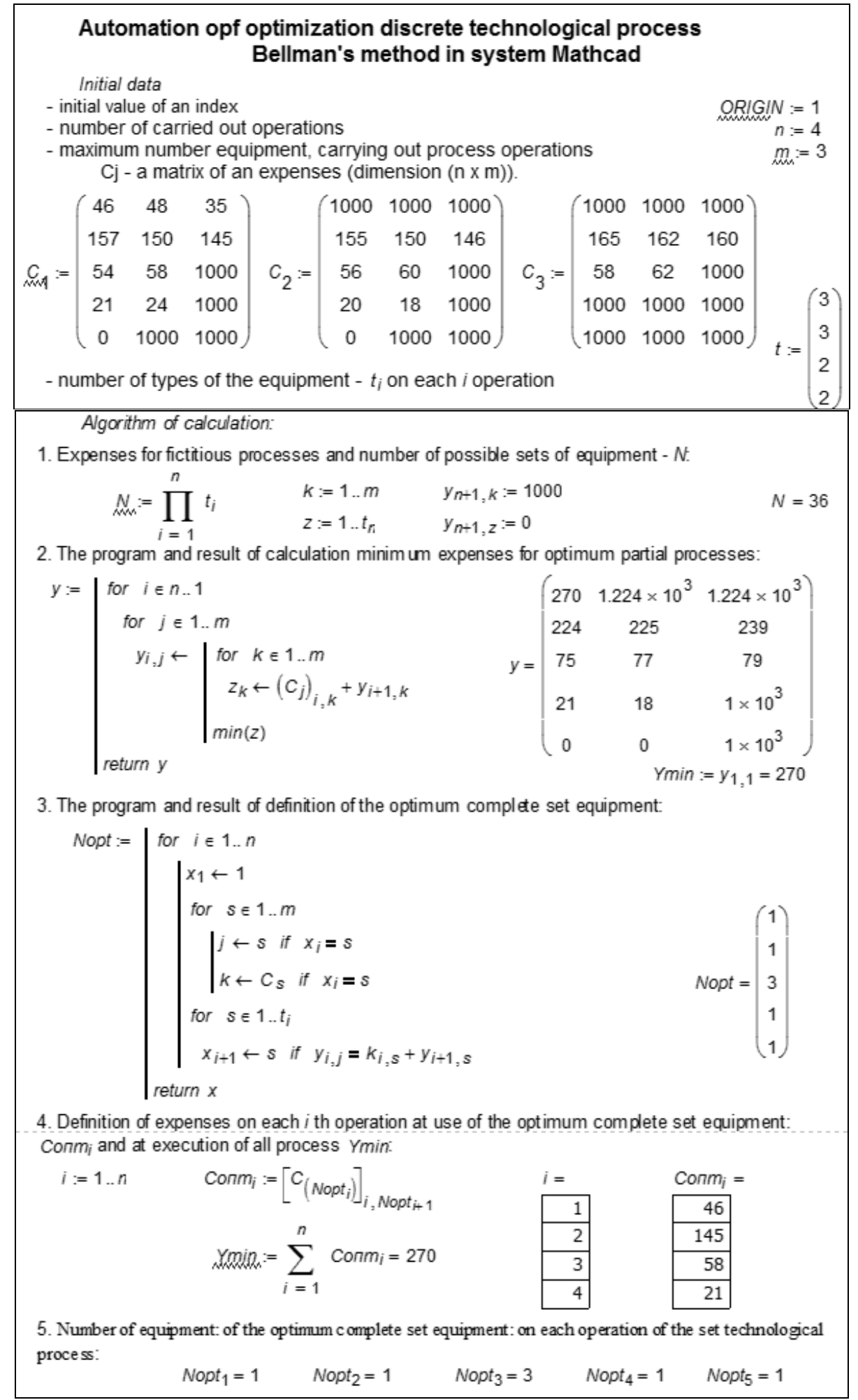

Fig. 3. Program start automation of optimization technological process 


\section{Conclusion}

The proposed procedure of structurally-parametrical optimization of technological process using Bellman's method and system Mathcad significantly decreases time and labour costs on execution of such calculations and efficiently executes investigations related with change of equipment parameters.

\section{References}

1. E. Kudryavtsev, Operations research in problems algorithms and programs (Moscow, Radio and communication, 1984)

2. E.Kudryavtsev, Mathcad 11. Russian Version Complete Guide (Moscow, DMK Press, 2005)

3. E. Kudryavtsev, Mathcad 2000 Pro.The symbolical and numerical decision of different problems (Moscow, DMK, 2001)

4. V. Ohorzin, Applied mathematics in system Mathcad (The manual, SPb., Lan Pub., 2009)

5. E. Kudryavtsev, Identification of Soil Excavation Operating Zone of Excavator Equipped with Backhoe Attachment (Procedia Engineering, 1642-1647, 2017)

6. E. Kudryavtsev, Automatic Calculation Techniques for Soil Digging Force Applied by Bucket Swing (Procedia Engineering, 1636-1641, 2017)

7. E. Kudryavtsev, Computer modelling, designing and calculation of elements of machines and mechanisms (Moscow, ASV, 2018)

8. E. Dijkstra, Discipline of programming (Moscow, Mir, 1978)

9. E. Dijkstra, K. Hoor, Structured Programming (Moscow, Mir, 1975)

10. R. Bellman, S. Dreyfus, Applied Dynamic Programming (Moscow, Mir, 1962)

11. R. Bellman, Dynamic programming (Moscow, Mir, 1957)

12. R. Bellman, S. Dreyfus, Applied Dynamic Programming (Moscow, Mir, 1962)

13. R. Bellman, Adaptive Control, a Guided Tour (Princeton. New Jersey, Princeton Univer. Press, 1961)

14. R. Bellman, Quarterly of Applied Mathematics, 16 (1), 87-90, (1957)

15. R. Ford, Jr., D. Fulkerson, Flows in Networks (Princeton University Press, 1962)

16. E.W. Dijkstra, Numerische Mathematik 1, 269-271 (1959)

17. J. Kruskal, Proc. Amer. Math. Soc. 7, 43-50 (1956)

18. H. Loberman, A. Weinberger, J. Ass. Comp. Mach. 4, 428- 437 (1957)

19. L. Ford, Network flow theory (Rand Corp. Paper, 1956)

20. C. Berger, Theorie des graphes et ses applications (Paris, Dunod K Press, 68-69, 2000) 